\title{
Evaluación, pruebas estandarizadas y procesos formativos: experiencias en escuelas secundarias del norte de México
}

\author{
DAVID MANUEL ARZOLA-FRANCO* \\ Centro de Investigación y Docencia de los Servicios Educativos \\ del Estado de Chihuahua, México
}

Recibido el 24-01-2016; primera evaluación el 13-04-2016;

segunda evaluación el 22-04-2016; aceptado el 07-05-2016

\section{Resumen}

En esta investigación se presenta un análisis de las políticas y procesos de aplicación de pruebas a estudiantes de escuelas secundarias en el Estado de Chihuahua, México. El objetivo está encaminado a comprender de qué manera estas prácticas guían o perfilan la dinámica de las escuelas, las prioridades del profesorado y el trabajo de los estudiantes. La información se recabó a través de entrevistas, grupos de enfoque y registros de observación. Los resultados señalan que el enfoque con el que se ha realizado la evaluación refuerza una cultura escolar que privilegia los resultados numéricos sobre los procesos formativos, se exalta la competitividad y el éxito individual por encima de la solidaridad y la empatía. Se contradice el enfoque de la evaluación plasmado en el currículo y las prácticas de evaluación en los salones de clase.

Palabras clave: política educativa, evaluación, pruebas estandarizadas, práctica docente.

\footnotetext{
* Profesor-investigador de tiempo completo del Centro de Investigación y Docencia de los Servicios Educativos del Estado de Chihuahua, México. Doctorado en Educación por la Universidad de Tijuana. Subdirector Académico del Centro de Investigación y Docencia (CID): 1999-2001; director del CID: 2001-2004; jefe de la Oficina de Vinculación del Departamento de Formación de Docentes de la Secretaría de Educación y Cultura de Estado de Chihuahua: 2001-2008; coordinador del Cuerpo Académico de «Política y Gestión en Educación» del CID: 2011-2016; socio del Consejo Mexicano de Investigación Educativa (COMIE) y de la Red de Investigadores Educativos Chihuahua (REDIECH); director de la Revista de Investigación Educativa de la REDIECH: ISSN: 2007-4336. Contacto: david.arzola@cid.edu.mx
} 
Evaluation, Standardized Tests and Training Processes: Experiences in Secondary Schools in North of Mexico

\section{Abstract}

In this research, an analysis of policies and implementation processes of testing high school students in the State of Chihuahua, Mexico is presented. The objective is aimed at understanding how these practices guide or outline the dynamics of schools, teachers and priorities of student work. The information was collected through interviews, focus groups and observation records. The results indicate that the approach that has made the assessment reinforces a school culture that favors the numerical results over the educational processes, competitiveness and individual success is exalted above solidarity and empathy. The evaluation approach embodied in the curriculum and assessment practices in classrooms is contradicted.

Keywords: educational policy, evaluation, standardized tests, teaching practice.

\section{Evaluación, pruebas estandarizadas e procesos formativos: experiencias em escuelas secundárias do norte de México}

\section{RESUMO}

Nesta pesquisa é apresentado uma análise das políticas e processos de implementação de testes de alunos do ensino médio no Estado de Chihuahua, México. O objectivo visa a compreensão de como essas práticas orientar ou delinear a dinâmica de escolas, professores e prioridades de trabalho do aluno. As informaçôes foram coletadas por meio de entrevistas, grupos focais e registros de observação. Os resultados indicam que a abordagem que tem feito a avaliação reforça uma cultura escolar que privilegia os resultados numéricos sobre os processos educativos, a competitividade eo sucesso individual é exaltado acima de solidariedade e empatia. A abordagem de avaliação incorporado nas práticas curriculares e de avaliação em salas de aula é contrariada.

Palavras-chave: política educacional, avaliação, testes padronizados, ensino. 


\section{De la eValuación en el aula a la eValuación como política pública, UN APUNTE HISTÓRICO A MANERA DE INTRODUCCIÓN}

La evaluación es consustancial a toda actividad humana, ya que de manera continua se requiere información que permita valorar las tareas que se han emprendido. Estas prácticas, de acuerdo a las concepciones y los propósitos que las respaldan, pueden tener la finalidad de acreditar, vigilar, regular, mejorar u optimizar las dinámicas que están en curso. En este sentido la evaluación puede utilizarse para medir, comprobar, comparar, emitir juicios o determinar méritos (Stufflebean \& Shinkfield, 2011), por lo tanto, no es una actividad lineal o unívoca, su enfoque depende siempre de las ideas o teorías que la sustentan y de los objetivos o metas que se hayan trazado (Wragg, 2003).

En la medida en que las sociedades evolucionaron también se diversificaron y sistematizaron los procedimientos evaluativos, de tal manera que actualmente se cuenta con sistemas con tal grado de desarrollo y sofisticación que han convertido a la evaluación en una disciplina, una industria y una profesión (Barrenechea, 2010; Tiana, 2009; Stufflebean \& Shinkfield, 2011).

En los centros escolares una parte esencial de proceso de enseñanza y aprendizaje está dedicada a esta tarea. La necesidad de buscar estrategias para la valoración de los estudiantes surgió a la par de los sistemas educativos formalmente constituidos, es decir con el advenimiento de los Estados nacionales en el siglo XIX (Díaz Barriga, 2006; Delval \& Lomelí, 2013; De Camilloni, 2010; Martínez Rizo, 2009; Rodríguez et al., 2006).

Aunque en su origen la evaluación tenía solo la pretensión de diagnosticar el nivel de los estudiantes que ingresaban a los centros escolares y registrar el progreso de los mismos durante su trayecto formativo (Delval \& Lomelí, 2013), con el paso del tiempo ha evolucionado para convertirse también en una política de interés público ampliamente promovida por gobiernos y organismos internacionales como la Unesco y la Organización para la Cooperación y el Desarrollo Económico (OCDE).

Este interés creciente por buscar información que vaya más allá del salón de clases, o de la escuela, está relacionado con la expansión de los sistemas educativos, la inversión progresiva de recursos públicos y la adopción de modelos fuertemente influidos por los procesos industriales que exigen controles para medir la calidad de los productos. Todo ello ha obligado a los gobiernos de la mayoría de los países a adoptar modelos de evaluación a gran escala mediante aplicaciones de pruebas estandarizadas.

Hay dos elementos destacados en la configuración de las políticas públicas para la evaluación educativa que dominan actualmente el escenario 
internacional, el primero tiene que ver con la masificación de la enseñanza, el segundo con las aportaciones de las teorías conductistas y el desarrollo de modelos de medición educativa cada vez más elaborados, producto de una racionalidad técnica (Casassus, 2010; Stufflebean \& Shinkfield, 2011).

Las pruebas objetivas se convierten en el instrumento por excelencia para evaluar el avance de los alumnos, en el mejor de los casos acompañados de otros recursos, pero la mayoría de las veces como elemento único y decisivo. A partir de entonces se registra una especie de isomorfismo entre evaluación y medición, y las pruebas dejan de ser un asunto local para convertirse en prácticas que en este momento tienen alcances nacionales o internacionales.

En las últimas décadas la investigación en el campo pedagógico, principalmente a partir de las aportaciones de las teorías constructivistas y socioculturales, reporta grandes avances en cuanto a la comprensión del aprendizaje, inteligencia humana, práctica educativa, culturas escolares y profesionales, el desarrollo y las necesidades de los estudiantes, que han permitido el impulso de propuestas curriculares y modelos de evaluación más amplios, abiertos y flexibles (Campos, 2011; Carretero, 2011; Coll, 2010) que contribuyen al "desarrollo de habilidades intelectuales de orden superior, así como la adquisición y promoción de valores y actitudes deseables, que hagan del individuo un sujeto autónomo, crítico, reflexivo, participativo, solidario y responsable de sus semejantes» (Moreno, 2002, pp. 32-33). Pero la literatura reporta también amplias brechas entre los planteamientos encaminadas hacia la evaluación formativa, las prácticas de evaluación de los docentes y los dispositivos de evaluación que se diseñan desde las instancias administrativas de alto nivel, ya que «la evaluación es una actividad compleja y sometida a discusión... plantea interrogantes acerca del rigor y la exactitud de la información recogida, la calidad de los análisis realizados o la objetividad de las conclusiones alcanzadas» (Tiana, 2009, p. 23).

En el caso de México, las políticas en materia de evaluación educativa vigentes tienen su origen en la década de 1990 con el Acuerdo Nacional para la Modernización de la Educación Básica (ANMEB, 1992), promovido desde la Secretaría de Educación Pública (SEP); el ANMEB es considerado un parteaguas para la educación mexicana y es el referente obligado para entender el estado actual de las políticas en materia educativa, que en mayor o menor medida han evolucionado a partir de estos planteamientos iniciales. Este acuerdo generó una dinámica en la que además de la renovación curricular y la formación de profesores, se incluía la creación de un sistema de estímulos económicos al desempeño docente y la aplicación de pruebas nacionales e internacionales para la evaluación de estudiantes. En este último aspecto 
hubo un cambio radical, ya que se transitó de un sistema cerrado en el que la información se guardaba con extremo recelo, a una condición en la que existen múltiples mecanismos de evaluación y los resultados se dan a conocer públicamente.

El Instituto Nacional para la Evaluación de la Educación (INEE) creado en 2002, es el ejemplo palpable de la forma en que estas políticas han ido emergiendo y se han consolidado. A partir de 2013 este Instituto se convierte en un órgano autónomo a través de una ley publicada en el Diario Oficial de la Federación que entre otras cosas pretende que las evaluaciones sean sistemáticas, integrales, obligatorias y periódicas (DOF, 2013).

$\mathrm{Al}$ analizar el panorama se pueden apreciar avances importantes en la creación de instituciones dedicadas a la evaluación educativa, además de la incorporación de México a programas de evaluación internacional, cuyas huellas se pueden rastrear en aplicaciones que tienen un arraigo considerable: en 1997 se comenzó a participar en el Programa Internacional de Evaluación de Estudiantes (PISA), a partir de ese mismo año México se incluyó los «Estudios Comparativos y Explicativos» promovidos por la Unesco a través de la Oficina Regional de Educación para América Latina y el Caribe. En el ámbito nacional se han desarrollado dos tipos de exámenes estandarizados para la educación básica: la Evaluación Nacional de Logro Académico en Centros Escolares (ENLACE), aplicada anualmente en el periodo 2006 a 2014, y los Exámenes para la Calidad y el Logro Educativos (EXCALE), que se aplican desde 2005. De estas experiencias, la que ha tenido una mayor resonancia en los centros escolares es ENLACE, por su aplicación universal en los niveles de primaria y secundaria, y por tener la peculiaridad de estar estrechamente ligada al sistema de estímulos económicos para los profesores.

Además de las evaluaciones descritas anteriormente, en algunas entidades federativas también se diseñan y aplican pruebas locales, algunas como réplica de los exámenes nacionales o bien dentro de la tradición de los concursos académicos. En el caso del estado de Chihuahua, en las secundarias generales y técnicas se llevan a cabo eliminatorias anuales por zona, por región y finalmente la etapa estatal. Además, en las secundarias técnicas se ha estado evaluando anualmente a los alumnos con el Examen Estatal (EXAEST).

En el desarrollo de los procesos de evaluación se pueden identificar dos rutas: la evaluación que realiza el docente como parte del proceso de enseñanza y aprendizaje, a la que se podría denominar interna, caracterizada por el uso de una gran variedad de recursos entre los que desde luego están incluidos los exámenes escritos. Y la evaluación que se lleva a cabo desde instancias externas que por lo regular se lleva a cabo a través de la aplicación pruebas estandarizadas. 
Cuando se trata esta última ruta por lo general hay una difusión amplia de los resultados, lo más común es la publicación de rankings en los que se destacan los puntajes más altos para entidades federativas, escuelas y estudiantes, pero no parece haber un interés especial en cuanto a las dinámicas que desencadenan las evaluaciones externas en los centros escolares, o sobre las percepciones que tienen al respecto los sujetos involucrados, como los supervisores, directores, docentes, jefes de enseñanza y los propios alumnos.

Las pruebas esconden tras de sí todo un proceso que no se agota ni se limita a la aplicación y la publicación de resultados, hay un antes y un después que permanece en la opacidad y del que evidentemente se pueden obtener lecciones para mejorar las dinámicas escolares y la misma evaluación. La presente investigación, realizada en ocho escuelas, ubicadas en cinco municipios del estado de Chihuahua, México, pretende indagar sobre los claroscuros que tienen políticas de esta naturaleza en la dinámica de los centros educativos, las prácticas de los docentes y la labor de los estudiantes de secundaria.

Las nociones de evaluación que sirven como base para el desarrollo de la presente investigación están ubicadas dentro del paradigma crítico, es decir se aborda a la evaluación como un fenómeno complejo, atravesada por intereses, valores, posiciones políticas (Lundgren, 2013; Tiana, 2009) ideológicas y prejuicios de diversa naturaleza, que pueden develar ciertas parcelas de la realidad a la par que ocultan otras.

Desde esta perspectiva, las prácticas de evaluación no tienen una posición neutral frente al objeto que estudian, forman parte de un escenario de debate, una arena política e ideológica en la que los involucrados pueden tener posiciones distantes e incluso antagónicas, toda evaluación «del rendimiento académico en alguna materia curricular concreta, en cualquier contenido escolar, refleja la concepción que del conocimiento y del mismo rendimiento tiene quien evalúa, más que el valor que tal contenido posee en sí» (Álvarez, 2001, p. 31).

Así como la evaluación no tiene sentido si se le separa del campo educativo, también es necesario evitar que se aísle de los contextos políticos, culturales, sociales y económicos que la sustentan, pues en ellos se pueden rastrear las raíces y concepciones que dan origen a determinadas prácticas. De ahí que en este trabajo se opte por concepciones que eviten el reduccionismo derivado de una "educación bancaria» (Freire, 2005, p. 81) para transitar hacia nociones abiertas, formativas y dialógicas, que ubiquen a la evaluación como parte consustancial de proceso educativo. 


\section{EL PROCESO DE INDAGACIÓN Y LAS DECISIONES METODOLÓGICAS}

En este proyecto estuvieron involucrados siete investigadores. Desde el punto de vista metodológico el equipo se propuso abordar el estudio desde una perspectiva etnográfica. Para el trabajo de campo se eligieron ocho escuelas secundarias ubicadas en cinco municipios del estado de Chihuahua, México: Juárez, Bocoyna, Cuauhtémoc, Hidalgo del Parral y Chihuahua capital, donde los investigadores permanecieron cuatro meses, trabajando tres días a la semana en horario regular, de 7:30 a 14:00 horas.

Las técnicas para recopilar información fueron: observación, entrevistas y grupos de enfoque. Aunque la etnografía no precisa de muestras estadísticamente representativas, se tomaron algunas decisiones que permitieran cubrir el espectro de escuelas secundarias del estado, los criterios básicos fueron: que incluyeran tanto secundarias técnicas como generales; que se eligieran escuelas de diferentes regiones: algunas ubicadas en los principales centros urbanos como Chihuahua y Juárez, pero también de la sierra como Bocoyna o ciudades pequeñas como Parral y Cuauhtémoc; y que se considerara a docentes y alumnos de los tres grados, así como a directores, subdirectores, supervisores, prefectos y asesores técnico-pedagógicos.

Durante la estancia se llevaron a cabo registros sistemáticos de las actividades, que fueron ampliados y convertidos en archivos electrónicos diariamente. Los docentes y directivos o administradores fueron entrevistados durante ese periodo, en promedio cada conversación duró una hora y media, se utilizaron guiones de entrevista y el apoyo de audio grabadoras y registros escritos; los sujetos se seleccionaron a partir de dos criterios: que tuvieran la experiencia suficiente para aportar información valiosa para el proyecto, y que se prestaran de manera voluntaria para participar.

Para los grupos de enfoque se seleccionaron alumnos de los tres grados y de las ocho escuelas; en cada grupo de enfoque participaron en promedio ocho estudiantes. Las audiograbaciones y registros de observación se transcribieron para ser procesados con el auxilio del programa Atlas Ti.

Las categorías de análisis que se trabajaron en la investigación fueron: organización y gestión, currículo y práctica educativa, formación y desarrollo profesional, y trayectorias laborales. El presente artículo se deriva de las categorías «currículo» y «organización y gestión». En total se entrevistaron 51 docentes, 4 subdirectores, 7 directores, 10 prefectos, 6 orientadores, 5 jefes de enseñanza y 3 supervisores. Además, se realizaron 14 grupos focales con la participación de 105 alumnos. 
De manera tradicional el trabajo etnográfico es una actividad que se desarrolla en solitario, el investigador se inserta en la vida de las comunidades con la intención de desentrañar las "estructuras de significación» (Geertz, 2006, p. 26) ocultas en la trama y urdimbre tejida por la cotidianeidad. En este caso el reto consistió en desarrollar un proyecto en el que se recuperan los elementos básicos de la etnografía a partir del trabajo en equipo, es decir desde el diseño hasta el análisis de la información las tareas se realizaron colectivamente, lo que implica la coordinación de un conjunto de tareas construidas a partir del diálogo y la búsqueda permanente de consensos. Esta labor incluye la formación misma del equipo de investigadores para crear las habilidades y la sensibilidad necesarias para detectar esos pequeños detalles e inflexiones que constituyen la cultura escolar: «viendo lo que pasa, escuchando lo que se dice, preguntando cosas; o sea recogiendo todo tipo de datos accesibles para poder arrojar luz sobre los temas que [se] han elegido estudiar» (Hammersley \& Atkinson, 2006, p. 15).

Gracias a la disposición de docentes, directores y administradores fue posible permanecer por más de cuatro meses en las instalaciones escolares «es un supuesto clave del trabajo que mientras mejor y por más tiempo conozcamos a la "gente», más profunda resultará la comprensión» (Agair, 1992, p. 135). Sin embargo, la entrada y permanencia en cada establecimiento demandó de diversas estrategias de negociación: algunos profesores se ofrecieron de inmediato para ser observados, la mayoría esperaron a que se les solicitara la autorización para entrar a las aulas y en solo se presentó el caso de un profesor que se negó a recibir a los investigadores.

De acuerdo con los intereses de cada investigador, el trabajo se desplegó abarcando y desarrollando diversas temáticas de las que han derivado, además del informe de investigación, un conjunto de trabajo individuales como el presente artículo.

Con la finalidad de sistematizar la información, cada testimonio incluido en este documento fue codificado a partir de la nomenclatura del programa Atlas Ti, por ejemplo: (Docente: 6:31; 682:697), indica que se trata de un profesor o profesora, el «6" se refiere al documento primario del que procede la información, el «31» muestra el código o categoría del que proviene la cita, y los números «682:697» son los renglones donde se ubica la cita en la base de datos, lo mismo se hizo con el reto de los sujetos que colaboraron en la investigación: subdirectores, directores, prefectos, orientadores, jefes de enseñanza, estudiantes y supervisores. 


\section{EVALUaCión ESTANDARIZADa CONTRA EVALUACIÓN FORMATIVA, UN DILEMA} PARA LOS PROFESORES DE SECUNDARIA

La aplicación de pruebas ocupa el centro de una buena parte de las preocupaciones de los colectivos de secundaria; a lo largo del ciclo escolar la mayoría de las acciones tienen como motivación o detonante algún tipo de proceso evaluativo, y resulta inquietante la manera en que el calendario escolar está saturado de este tipo de actividades. Un profesor expresa: «Yo les dije a los muchachos: ahí está el temario y échele ganas... y ahora nos exigen dos exámenes parciales, los bimestrales, los concursos académicos y los regionales si llegamos allá... luego ENLACE» (Docente: 1:534; 3027:3034).

Una parte sustancial de la actividad escolar está supeditada a estas prácticas, que forman parte de una serie de políticas educativas de diverso alcance que son impuestas a las instituciones, y que como todo proceso estandarizado tiene un paralelo con las actividades industriales: aplicación en serie, estudiantes formados en el patio, la arenga, entrada a los salones de clase, pase de lista, asignación de números, tiempos cronometrados, periodos de descanso entre una aplicación y otra, los resultados como producto y el discurso de la calidad.

La obligación de participar en la evaluación ha generado preocupaciones por la imagen que las escuelas proyectan, comparación entre unos centros y otros, y énfasis en las puntuaciones. Aunque en el taller informativo ENLACE se señala que "las mejores escuelas no necesariamente son las que obtienen mejores resultados, sino aquellas que aportan más a sus estudiantes» (SEP, 2010, p. 63), en los hechos estos puntajes, analizados al margen del contexto educativo, tienen un peso enorme en el ánimo de profesores y directivos, de tal manera que el resultado se convierte en un fin en sí mismo, lo que evidentemente va a contracorriente con el enfoque enunciado en el currículo:

La evaluación es un proceso continuo de obtención de información que no se reduce a la aplicación periódica de pruebas. Por lo cual es necesario, dentro de lo posible, eliminar las actividades que no promueven el aprendizaje, tales como dedicar tiempos especiales a preparar a los alumnos para la resolución de exámenes, o proporcionarles "guías de estudio" que solo sirven para memorizar información y pasar un examen (SEP, 2007, p. 53).

Los colectivos escolares reciben un mensaje confuso cuando simultáneamente se exigen resultados numéricos y una evaluación formativa. La elección resulta obvia, los números se pueden mostrar, exhibir, presumir, es la forma en que regularmente se tasa socialmente a las escuelas. Su prestigio está fincado en los resultados de las evaluaciones que se aplican durante el 
ciclo escolar, aunque en pocas ocasiones se destaca el problema que implica medir de manera igual a los desiguales, "se robustece así el poder de quienes tienen más, sirviendo también como elemento discriminatorio, promotor de injusticia social» (Caviedes, 2013, p. 9). Por otra parte, los resultados se esgrimen como un arma política para cuestionar la tarea del profesorado, en este sentido el examen, «con su aplicación masiva, ha pasado de ser un instrumento del docente, para convertirse en un instrumento contra el docente» (Padilla Magaña, 2009, p. 45).

Este afán por los resultados es justificable si se considera la presión social que existe para mejorar las cifras y puede incluso generar obsesiones entre el personal de algunos centros educativos cuando las estrategias se enfocan en el entrenamiento de los estudiantes para contestar reactivos (Koretz, 2005), así como en el endurecimiento de los criterios de evaluación:

Aquí la escuela es muy rígida, un seis de nosotros en otra escuela equivale a un ocho o un nueve... Considero yo que las normas de evaluación son muy blandas como lo marca la reforma, en todas partes ¿ENLACE qué mide?... conocimiento... ¿El examen de admisión?... conocimiento (Director: 5:25; 458:492).

Con lo anterior se colige que predomina la evaluación como producto, por encima de la idea de la evaluación como proceso, «este tipo de evaluación incentiva a los docentes a que dediquen gran parte de su tiempo en la preparación exclusiva para esas evaluaciones» (Barrenechea, 2010, p. 3). El discurso que se reproduce a continuación fue dirigido a un grupo de primer grado como parte del entrenamiento para estudiantes:

Vengo a pedirles compromiso... que den su máximo esfuerzo... ¿qué significa? No faltar porque la norma dice que si falta el 5\% de los alumnos se anula, así que no se vale enfermarse... Lo segundo, que den lo mejor, échenle ganas. $\mathrm{Si}$ usted está en el concurso estatal es porque su maestro le echó ganas. El lunes temprano vengo a pasar lista, nueve grupos tienen examen a las 8:20, más tarde tienen examen de bimestre (Subdirector: 1:204; 147:1158).

Estos resultados son muy importantes para la proyección de los establecimientos, entre las estrategias para preparar a los alumnos, se destacan la aplicación de exámenes de repaso y prácticas de reforzamiento con ejercicios relacionados con la materia en la que van a participar:

Se puede observar cómo de manera consciente e inconsciente se han estado implementando actividades como resolución de pruebas similares, buscando la familiarización a las mismas por parte de los alumnos, los repasos de temáticas 
abordadas en exámenes anteriores en tiempos de clase, etcétera. Con ello se da prioridad a resultados ligados con incentivos económicos y con la etiquetación del docente ante las autoridades, organizaciones públicas y privadas con injerencia en la educación (Bautista Sánchez, 2015).

Como ejemplo de estas prácticas, en una de las escuelas visitadas se citó al personal para informarles que los profesores de educación física, artes, tecnologías y tutoría, cederían su tiempo para que los alumnos se prepararan para la prueba (Observación: 1:130; 156:167), además los docentes cuyos grupos iban a participar también dedicaron su tiempo para reforzar contenidos.

Si la eficacia de la instrucción se juzga desde instancias externas solo a partir de las respuestas en los exámenes, surge en los profesores la tentación de preparar a los alumnos para lo que se les va a exigir en las pruebas; tanto más si las consecuencias de las malas notas recaen personal y directamente en el profesor (Rodríguez et al., 2006).

La estrategia básica consiste en aplicar un examen y contestarlo en binas o equipos y posteriormente comparar las respuestas:

...les empezamos a dar copias extras empezamos a ver reactivo por reactivo, los muchachos entran en una dinámica que se emocionan por ganar y todas esas clases extra que se les da, digamos que de repaso, pues ellos van reponiendo todo lo que no han aprendido (Docente: 9:122; 1980:1992).

Resulta evidente que en algunas escuelas hay gran entusiasmo por participar, se alienta el espíritu competitivo en los estudiantes y se invierte un tiempo considerable en su entrenamiento, lo importante en estos casos es el orgullo de ser el mejor:

Ellos van siempre con la mentalidad hacia ganar... Mire, el profesor de matemáticas de tercer grado no ganó para ir al estatal... el maestro, me dijo: iya tiene el grupo que me va a tocar el año que entra? porque voy a trabajar desde ahorita... se empieza a trabajar desde septiembre para concursar en marzo o en abril (Director: 5:19; 329:345).

Aunque son prácticas con cierto arraigo y aceptación en las escuelas, hay profesores que con sentido crítico advierten los riesgos implícitos que acarrea la competitividad cuando conduce al abandono del sentido formativo de la educación, enfatizan particularmente los problemas que acarrean los concursos académicos:

Las tensiones vienen porque tiene uno que ganar, eso también es un desagrado que tengo... a mí me toca concursar y no termino de ver mis contenidos porque voy en cuarto bimestre y el examen de concurso es hasta tercero y me 
dicen deja cuarto bimestre, déjalo para después porque tienes que prepararte con los primeros tres (Docente: 6:31; 682:697).

El aspecto competitivo de estas actividades tiene dos caras: la de la victoria, es decir escuelas que por lo regular obtienen buenos resultados, que sirven para abonar su prestigio y el orgullo de pertenecer a un grupo privilegiado; y la de la derrota, escuelas que a pesar del esfuerzo no logran colocarse en alguno de los lugares importantes, lo cual genera frustraciones, resentimientos, discordias, rivalidades y la sospecha de ilegitimidad e inequidad en los procedimientos y sus resultados:

En lo que a veces no estoy de acuerdo es la forma en cómo se dan creo que siempre salimos enojados ¿¿no? el único que sale contento siempre es el ganador y el que pierde sale pues enojado (Docente: 10:14; 255:286).

Le voy a decir que aquí están todos en contra de nuestra escuela... de diecisiete materias que hubo en los concursos académicos nuestra escuela se llevó doce y nada más quedaron cinco materias para repartirlas en veinte escuelas ... por nuestra parte no hay agresión hacia las otras escuelas, pero de las otras escuelas para con nosotros sí (Director: 5:18; 308:328).

Quizá el problema más grave está en el hecho de que estas prácticas someten a las escuelas a una competencia que en ocasiones tiene tientes darwinianos, se privilegia la competitividad entre instituciones cuyos estudiantes provienen de estratos sociales, culturales y económicos tan diversos que no puede haber punto de comparación posible. Con estas prácticas las escuelas contribuyen a ensanchar la brecha de la inequidad ya que se "reproducen, multiplican e incluso intensifican las diferencias de entrada al sistema» (Blanco, 2009, p. 1020), los más aptos son los que se destacan, reciben reconocimientos, incluso estímulos económicos para el personal docente. «Se busca así crear a un estudiante con un perfil orientado a ganar a cualquier costo, a ser el mejor, a dejar atrás a los demás» (Aboites, 2009, p. 5).

Por otra parte el ubicar y señalar públicamente a las escuelas con indicadores educativos desfavorables con la finalidad de implementar programas para la mejora, parece tener un efecto contraproducente puesto que se convierte en un estigma para estos centros educativos, que a sus condiciones de origen se agrega el descrédito de ser escuelas «focalizadas», lo cual aleja a los estudiantes que tienen otras opciones y deja a quienes no tienen alternativas en la difícil situación de cargar con el desprestigio:

Estamos bajísimos, de aquí de la zona, somos la número dieciocho de dieciocho y en el nivel estatal bajamos todavía más, cerca del lugar ochenta... La escuela 
desgraciadamente por su historia y las condiciones en las que se ha estado haciendo el trabajo es vista como el patito feo... también la gente se siente desvalorada y poco motivada cuando empieza un programa de focalización de escuelas porque salimos ubicados en ENLACE varios años (Director: 29:103; 140:144).

La competencia implica que cuando hay un ganador tiene que haber muchos perdedores, solo los que tienen mayor capacidad de adaptación, más recursos y mejores condiciones pueden sobresalir. El sistema mismo, al enfatizar de manera desproporcionada los resultados numéricos sin considerar el contexto social y económico que rodea a las escuelas, privilegia la competitividad por encima de los sentimientos de solidaridad (Blanco, 2009). Aunque esta perspectiva tiene un fuerte arraigo en la cultura escolar:

Mira, creo yo que en esta vida estamos llenos de retos y la vida es una competencia siempre... aunque sean alumnos de secundaria deben de ir entendiendo que la vida así va a ser, yo estoy de acuerdo en que hay que competir ¿Y cómo le puedes hacer?... destacándome de todas las demás (Director: 8:16; 234:261).

Quienes obtienen la victoria ganan confianza, seguridad y respeto, quienes pierden se quedan con una imagen socavada de sí mismos. Si a esto se le agrega que provienen de zonas marginadas y de contextos socialmente desfavorecidos la escuela probablemente esté contribuyendo a acentuar más esas diferencias, además con la estigmatización surge la rivalidad y el resentimiento. Estos recelos están presentes también al interior de las escuelas triunfadoras puesto que el estímulo económico derivado de los resultados de ENLACE provoca animadversiones entre el personal:

Nosotros obtuvimos el segundo lugar a nivel estatal en ENLACE, en español y matemáticas, y según esto el incentivo a nivel nacional viene para toda la escuela, para todos los maestros y ahí nosotros estuvimos un poquito en desacuerdo por la razón de que muchos maestros no le echan las mismas ganas (Docente: 15:218; 78:82).

Sí, es estresante cuando a uno la evalúan porque tengo que acabar mi programa... cuando otro maestro no está evaluado no le importa y recibe lo mismo o más que uno, cómo es posible que le dieron a los tres coordinadores, director y subdirector... cómo es posible que si yo tengo siete grupos y en la tarde nada más uno nos toque lo mismo ¿Cuál es el estímulo? (Docente: 20:430; 2851:2869).

Los efectos de estas prácticas tienen también repercusiones en el ánimo y la autoimagen de los estudiantes, se reproducen a continuación testimonios de dos grupos de alumnos: 
Grupo 2

Alumno A: No... nosotros no participamos en el concurso.

Investigador: ¿por qué no participaron?

Alumno A: Porque somos un grupo muy feo.

Alumno B: Somos de bajo nivel académico.

Investigador: ¿Y ustedes que piensan de eso?

Alumno A: Pues que nos hacen pasar como que no tenemos la capacidad, que somos el único salón que no tenemos la capacidad.

Alumno B: - Pero, por ejemplo Perla es muy inteligente hay varias que son muy inteligentes no digo que somos porque no lo soy $(2: 41 ; 704: 712)$.

\section{Grupo 3}

Investigador: ¿¿Su grupo participó en los concursos académicos?

Alumnos: No...

Alumno D: - Pues es que sí hay buenos alumnos que sacan bien y todo, por ejemplo Elías, Johana, si hay personas que sacan buenas calificaciones cuando quieren, entonces, ya no los cuentan... pero rara vez los de la tarde.

Alumno A: -En la tarde son los desechos de la mañana, somos... no sé, los que van hasta atrás y no les toca nada, somos basura para ellos.

Alumno C: No son celos verdad.

Alumno D: Es racismo, no nos quieren... (4:19; 634:722).

Este afán por la competencia tiene repercusiones importantes en la formación y construcción de ciudadanía ya que «la competencia no sirve para crear un ambiente de unión y solidaridad [...] justifica las diferencias en salud, educación, ingreso económico y calidad de vida» (Aboites, 2009, p. 6). El énfasis en los resultados relega a un papel marginal los aspectos esencialmente formativos del proceso de enseñanza y aprendizaje: «hay riesgo de que el trabajo de escuelas y maestros se oriente en función de la prueba, descuidando aspectos importantes del currículo no cubiertos por ella» (Martínez Rizo, 2009, p. 8).

\section{LAS POLÍtTICAS DE EVALUACIÓN COMO UN PROCESO CONTRADICTORIO, REFLEXIONES A MANERA DE CIERRE}

Se detectan profundas contradicciones entre el discurso oficial plasmado en el currículo y la aplicación de instrumentos estandarizados como la prueba 
ENLACE y otros diseñados por las autoridades educativas estatales y regionales, puesto que en el primer caso se pretende privilegiar el enfoque formativo de la evaluación, mientras en el segundo las aplicaciones y la publicación de resultados derivaron en una competencia desleal y en análisis superficiales y maniqueos a nivel de la opinión pública:

[...] se muestran y enfatizan las diferencias entre estados, entre escuelas privadas y públicas, entre escuelas comunitarias y generales, entre secundarias técnicas, generales y telesecundarias. Además, para fomentar la idea de que lo que importa es el triunfo individual, se destaca y premia a las escuelas y a los estudiantes que a nivel nacional obtuvieron los puntajes más altos. En el fondo, detrás de la estrategia de evaluar para individualizar y competir está la tesis de que la manera más eficaz de mejorar la educación es saturarla de los valores, prácticas e ideología del mercado (Aboites, 2009, p. 5).

Quizá el aspecto más cuestionable de estas aplicaciones fue el hecho de ligarlas con el programa de estímulos económicos al desempeño docente, lo cual contribuyó a distorsionar aún más los resultados ya que los docentes más que ocuparse por mejorar el aprendizaje de los alumnos se preocupan por sus ingresos, el puntaje de los alumnos adquiere un valor monetario, con lo cual se desplegaron un conjunto de estrategias enfocadas al adiestramiento de los estudiantes para contestar los exámenes.

Estas aplicaciones han provocado también un fenómeno creciente de comparación entre buenas y malas escuelas, buenos y malos docentes, buenos y malos alumnos; el uso discrecional de resultados, al margen del contexto social en el que están inmersos, ha servido para tasar socialmente a estos actores y escuelas. Esto tiene profundas implicaciones en la autoimagen de estudiantes y docentes quienes se sienten excluidos o relegados.

En demérito de una verdadera formación para los estudiantes, con el uso y abuso de las pruebas estandarizadas, se privilegia la competencia, el enfrentamiento, la rivalidad, la disputa, con altas dosis de discriminación hacia las escuelas menos favorecidas, en menoscabo de las competencias es decir la movilización de recursos para enfrentar situaciones problemáticas (Perrenoud, 2004) y desarrollar «estrategias metacognitivas» (CERI, 2005, p. 50) para el aprendizaje autónomo.

Estas prácticas están acompañadas de una presión permanente en los centros educativos y en buena medida definen la dinámica institucional ya que obligan a profesores y directivos a implementar estrategias ex profeso para estar a la altura de las expectativas, con el riesgo que ellos mismos advierten de marginar los aspectos esenciales del currículo y lo que es más grave, que el estudiante y su 
formación integral queden en segundo plano. El énfasis en el producto como un fin en sí mismo y el ganar como una meta, elude el propósito enunciado de evaluar para mejorar procesos de enseñanza y aprendizaje, para realimentar las prácticas y buscar alternativas para dotar al estudiante de las competencias básicas que le permitan construir las bases para una vida digna.

ENLACE, y otras pruebas que se aplican en la entidad, no son necesariamente el origen de muchas de las prácticas y problemas descritos, hay evidencias de dinámicas de esta naturaleza con un profundo arraigo en los centros escolares de este nivel educativo; sin embargo, la implementación de estas políticas han contribuido a potenciar este afán competitivo, fueron asimiladas por una cultura en la que se premia el acierto y se castiga el error. Desde el plano docente, hay evidencias de distanciamiento entre los aportes teóricos de avanzada y las prácticas de evaluación (Moreno, 2002), asimismo se encuentran profundas contradicciones en el discurso oficial, que por un lado impulsa el enfoque por competencias y la necesidad de una evaluación formativa que atienda las necesidades de los alumnos y su contexto, pero al mismo tiempo implementa mecanismos de evaluación que tienden a etiquetar a los estudiantes, a los profesores y las escuelas.

A partir del ciclo escolar 2014-2015 la prueba ENLACE fue suspendida por la SEP, y el INEE está trabajando para el diseño de nuevas propuestas, no obstante, ocho años de aplicación de este y otros instrumentos similares han dejado importantes lecciones:

Si comenzamos por los beneficios resulta obvio que estos mecanismos de evaluación pueden contribuir a transparentar las condiciones del sistema educativo. Esta información coadyuva de alguna manera en la toma de decisiones informadas y en la construcción de un ambiente de autocrítica y de apertura hacia la participación social. Sin embargo, estas aplicaciones han permitido visualizar los sesgos que acarrea el uso de estos instrumentos cuando se utilizan con propósitos para los que no fueron diseñados. Si bien los datos que se obtienen con una prueba estandarizada dan una visión panorámica de la problemática educativa nacional, regional o local, en realidad ayudan muy poco cuando se pretende ubicar las causas de dichos problemas, además no sirven "para casi nada cuando hubo que diagnosticar las necesidades y valorar los avances de los niños menos favorecidos» (Stufflebean \& Shinkfield, 2011, p. 38).

Para las escuelas, las pruebas nacionales o internacionales solo pueden ser un referente o indicador que, acompañado de otros elementos relacionados con el contexto inmediato (Darling-Hammond \& Snyder, 2000), y con una amplia participación de la comunidad, sirve como base para la identificación de los problemas y el planteamiento de estrategias para la mejora de los centros 
educativos, este cuidado y vigilancia cercana de los procesos implica desde luego altos niveles de autonomía, situación de la que los centros escolares mexicanos están muy alejados.

Las tensiones en las prácticas de evaluación cuando se intenta conciliar los discursos con las acciones, son el reflejo de un problema estructural derivado de un sistema educativo altamente centralizado y con tendencias homogeneizantes, paradójicamente inserto en un mundo en el que se exalta el respeto a la diversidad, la horizontalidad y la apertura democrática. Aunque la misma SEP habla de la necesidad de expandir las posibilidades de autonomía de las escuelas, en realidad despliega acciones para conservar los mecanismos de control que le permiten tomar decisiones a nivel central en torno al currículo, los materiales educativos, el calendario escolar, ingreso y permanencia de profesores, estímulos al desempeño y por supuesto los mecanismos de evaluación de docentes y alumnos.

\section{REFERENCIAS BIBLIOGRÁFICAS}

Agair, M. (1992). Hacia un lenguaje etnográfico. En C. Geertz y J. Clifford (coords.), El surgimiento de la antropología posmoderna (pp. 117-137). Barcelona: Gedisa.

Aboites, H. (2009). La prueba Enlace: una pésima medicina para un sistema educativo enfermo. La necesidad de otra evaluación. Documento PDF, 3 de mayo de 2009. Recuperado el 3 de agosto de 2014 de http://132.248.35.1/ ec/Foroed/Aboites.pdf

Álvarez Méndez, J. M. (2001). Evaluar para conocer, examinar para excluir. Madrid: Morata.

Barrenechea, I. (2010). Evaluaciones estandarizadas: seis reflexiones críticas. Archivos Analiticos de Políticas Educativas, 18(8), 1-25.

Bautista Sánchez, E. J. (2015). La evaluación mediante pruebas de gran escala en México. Revista Iberoamericana para la Investigación y el Desarrollo Educativo, 5(10), 1-15. https://doi.org/10.23913/ride.v5i10.9

Blanco, E. (2009). La desigualdad de resultados educativos. Aportes a la teoría desde la investigación sobre eficacia escolar. Revista Mexicana de Investigación Educativa, 14(43), 1019-1049.

Campos, B. (2011). Mejorar la práctica educativa. Herramientas para optimizar el rendimiento de los alumnos. Madrid: Wolters Kluwer.

Carretero, M. (2011). Constructivismo y educación. Buenos Aires: Paidós.

Casassus, J. (2010). Las reformas basadas en estándares: un camino equivocado. Educare, 5(9), 85-107. 
Caviedes, J. F. (2013). Las evaluaciones estandarizadas desde una visión freireana. Revista Elecrónica de Educación, 7(1), 369-382.

CERI (2005). Formative assessment. Improving learning in secondary classrooms. París: OECD-Centre for Educational Research and Innovation. Recuperado de https://www.oecd.org/edu/ceri/35661078.pdf

Coll, C. (2010). Aprendizaje escolar y construcción del conocimiento. México: Paidós.

Darling-Hammond, L. \& Snyder, J. (2000). Authentic assessment of teaching in context. Teaching and Teacher Education, 16, 523-545. https://doi. org/10.1016/S0742-051X(00)00015-9

De Camilloni, A. (2010). La validez de la enseñanza y la evaluación. En R. Anijovich, A. de Camilloni, G. Cappelletti, J. Hoffmann, R. Katzkowicz y L. Mottier López, La evaluación significativa (pp. 23-42). Buenos Aires: Paidós.

Delval, J. \& Lomelí, P. (2013). La educación democrática para el siglo XXI. México: Siglo XXI.

Diario Oficial de la Federación (1992). Acuerdo Nacional para la Modernización de la Educación Básica. México: SEP.

Díaz Barriga, Á. (2006). Las pruebas masivas, análisis de sus diferencias técnicas. Revista Mexicana de Investigación Educativa, 11(29), 583-615.

DOF (2013, 11 de septiembre). Ley del Instituto Nacional para la Evaluación de la Educación. Recuperado el 4 de noviembre de 2014, de DOF - Diario Oficial de la Federación: http://www.dof.gob.mx/nota_detalle.php?codigo $=5313842 \&$ fecha $=11 / 09 / 2013$

Freire, P. (2005). Pedagogy of Indignation. Boulder: Paradigm.

Geertz, Clifford (2006). La interpretación de las culturas. Barcelona: Gedisa.

Hammersley, M. \& Atkinson, P. (2006). Etnografia, métodos de investigación. Barcelona: Paidós.

Koretz, D. M. (2005). Alignment, high stakes, and the inflation of test scores. CSE Report 655. Los Angeles: University of California.

Lundgren, P. (2013). PISA como instrumento político, la historia detrás de la creación del programa PISA. Profesorado, 17(2), 15-29.

Martínez Rizo, F. (2009). La evaluación de la calidad de los sistemas educativos: propuesta de un modelo. En E. Martín y F. Martínez Rizo (coords.), Avances y desafios en la evaluación educativa (pp. 27-39). Madrid: OEI/ Fundación Santillana.

Moreno, T. (2002). Cultura profesional del docente y evaluación del alumnado. Perfiles Educativos, 24(95), 23-35. 
Padilla Magaña, R. A. (2009). Exámenes masivos internacionales y nacionales. ¿Encuentros o desencuentros? Perfiles Educativos, 31(123), 44-59.

Perrenoud, P. (2004). Diez nuevas competencias para enseñar. Barcelona: Grao.

Rodríguez, T., Álvarez, L., Gonález-Castro, P., González-Pineda, J. A., Muñiz, J., Nuñez, J. C. \& Soler, E. (2006). La evaluación de aprendizajes. Madrid: CCS Editorial.

SEP (3 de abril de 2014). Evaluación Nacional del Logro Académico en Centros Escolares. Recuperado el 19 de noviembre de 2014, de ENLACE: http:// www.enlace.sep.gob.mx/

SEP (2007). Plan de estudios de educación secundaria 2006. Segunda edición. México: Secretaría de Educación Pública.

SEP (2010). Taller informativo educación básica y media. Portal Enlace, consultado el 10 de noviembre de 2013 en: http://www.enlace.sep.gob.mx/ $\mathrm{gr} /$ ?p=quees

Stufflebean, D. \& Shinkfield, A. (2011). Evaluación sistemática, guía teórica y práctica. Madrid: Paidós/Ministerio de Educación y Ciencia.

Tiana, A. (2009). Evaluación y cambio educativo: los debates actuales sobre las ventajas y los riesgos de la evaluación. En E. Martín y F. Martínez Rizo (coords.), Avances y desafios en la evaluación educativa (pp. 17-26). Madrid: OEI/Fundación Santillana.

Wragg, E. (2003). Evaluación y aprendizaje en la escuela primaria. Barcelona: Paidós. 